\title{
A Noise Investigation of Tunnel-Diode Microwave Amplifiers*
}

\author{
A. YARIV $\dagger$, MEMBER, IRE, AND J. S. COOK $\dagger$, MEMBER, IRE
}

\begin{abstract}
Summary-An analysis and derivation of the noise figure of a tunnel-diode microwave amplifier are presented. The agreement between the measured noise figure and the theoretical results is an indirect check on the existence of full shot noise in germanium tunnel diodes at microwave frequencies. The limiting noise temperature of the amplifier is $e I_{0} R / 2 k$, and can be approached by using diodes with small $(R C)$ products in which the extreme overcoupling (load mismatch) and high gain can be achieved simultaneously.
\end{abstract}

\section{INTRODUCTION}

$\Lambda^{M}$ MPLIFIERS utilizing tunnel diodes were first reported by Chang ${ }^{1}$ at frequencies up to $80 \mathrm{Mc}$. He also suggested that the noise contribution of the diode was due to the shot noise accompanying the tunneling process. This suggestion was confirmed by Lee and Montgomery, ${ }^{2}$ who showed by measurements at $5 \mathrm{Mc}$ that full shot noise was indeed generated by the tunnel diode. Tiemann ${ }^{3}$ has reported results similar to those of Lee and Montgomery, obtained from noise measurements at $500 \mathrm{kc}$ on germanium diodes.

Approximate noise analyses of tunnel-diode amplifiers were given by Sommers, et al., ${ }^{4}$ Tiemann, ${ }^{3}$ and Hines and Anderson. ${ }^{5}$ The simplified equivalent circuits used were sufficient to derive the limiting noise behavior for very high gain and extreme overcoupling. Yariv, et $a l .{ }^{6}$ reported the operation of a tunnel-diode amplifier at microwave frequencies and measured its noise figure.

In this paper, the details are given of the noise analysis which led to the noise temperature formula stated by Yariv, et al. ${ }^{6}$ This analysis is based on an "exact" equivalent circuit, and its results are useful in the intermediate range of small or medium gain as well as in the limiting case described above. The theoretical results are compared with the noise measurements.

\section{The Physical Sources of Noise}

In addition to the omnipresent noise generated by the ohmic losses in the spreading and contact resistance of the diode and the surrounding microwave structure, we

* Received by the IRE, October 3, 1960.

† Bell Telephone Labs., Inc., Murray Hill, N. J.

1 K. K. N. Chang, "Low-noise tunnel-diode amplifier," Proc. IRE (Correspondence), vol. 47, pp. 1268-1269; July, 1959.

${ }^{2}$ C. A. Lee and H. C. Montgomery, "Determination of forward and reverse tunneling currents in Esaki diodes by shot noise measurements," Bull. Am. Phys. Soc., vol. 3, p. 160; March 21, 1960.

3 J. J. Tieman, "Shot noise in tunnel diode amplifiers," Proc. IRE vol. 48, pp. 1418-1423; August, 1960.

${ }^{4}$ H. S. Sommers, Jr., et al., "Tunnel diodes for low noise amplification," 1959 IRE WESCON CONVENTION RECORD, pt. 3, pp. 3-8.

${ }_{5} \mathrm{M}$. E. Hines and W. W. Anderson, "Noise performance theory of Esaki (tunnel) diode amplifiers," Proc. IRE (Correspondence), vol. 48, p. 789; April, 1960.

6 A. Yariv, et al., "Operation of an Esaki diode microwave amplifier," Proc. IRE (Correspondence), vol. 48, p. 1155; June, 1960. must consider the shot noise accompanying the tunneling process. ${ }^{7}$ There are two separate and independent tunneling currents, one consisting of electrons tunneling from the $N$ to the $P$ side of the junction, which we denote by $i_{1}$, and a second current, $i_{2}$, made up of electrons crossing the junction in the opposite direction. The net measurable dc current is $I_{0}=\left|i_{1}\right|-\left|i_{2}\right|$. Since the currents $i_{1}$ and $i_{2}$ are uncorrelated, the total shot noise ${ }^{8}$ is represented by a current generator of mean-square amplitude,

$$
\overline{i^{2}}=2 e\left(\left|i_{1}\right|+\left|i_{2}\right|\right) B
$$

where $e$ is the electronic charge and $B$ is the bandwidth in cycles per second in which the noise is considered. When the diode is biased so as to operate in the negative resistance region (see Fig. 1 ), the current $i_{2}$ is negligibly small and the approximation

$$
I_{0} \approx\left|i_{1}\right|+\left|i_{2}\right|
$$

may be used.

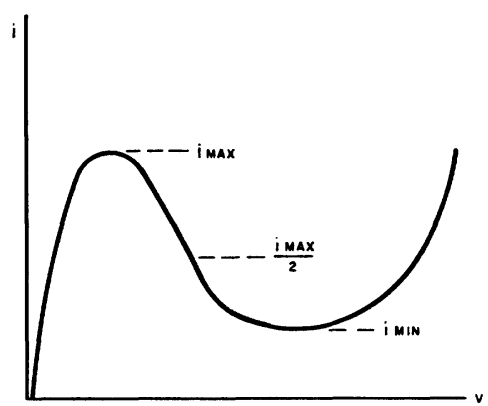

Fig. 1-A typical voltage-current characteristic curve of an Esaki diode.

\section{The Equivalent Circuit}

The amplifier constructed for the noise experiment consists of a germanium diode mounted in a single port cavity which is coupled to the load and the source by a low-loss circulator as shown in Fig. 2, the coupling being continuously variable. The equivalent circuit of this amplifier is shown in Fig. 3. The diode is represented by a negative resistance $-R$, shunted by a capacitance $C$,

${ }^{7} \mathrm{~L}$. Esaki, "New phenomenon in narrow germanian $p-n$ junctions," Phys. Rev., vol. 109, pp. 602-603; June, 1958.

${ }^{8}$ See, for instance, J. R. Pierce, "Physical sources of noise," Proc. IRE, vol. 44, pp. 601-608; May, 1956. 


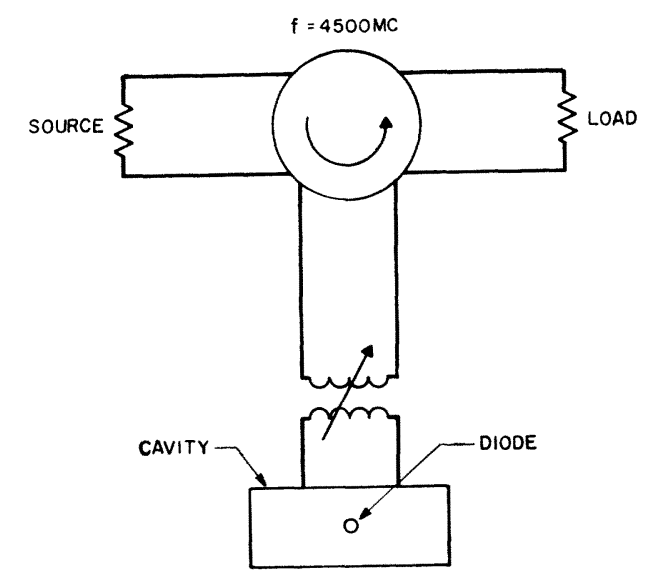

Fig. 2-A schematic view of the Esaki diode amplifier.

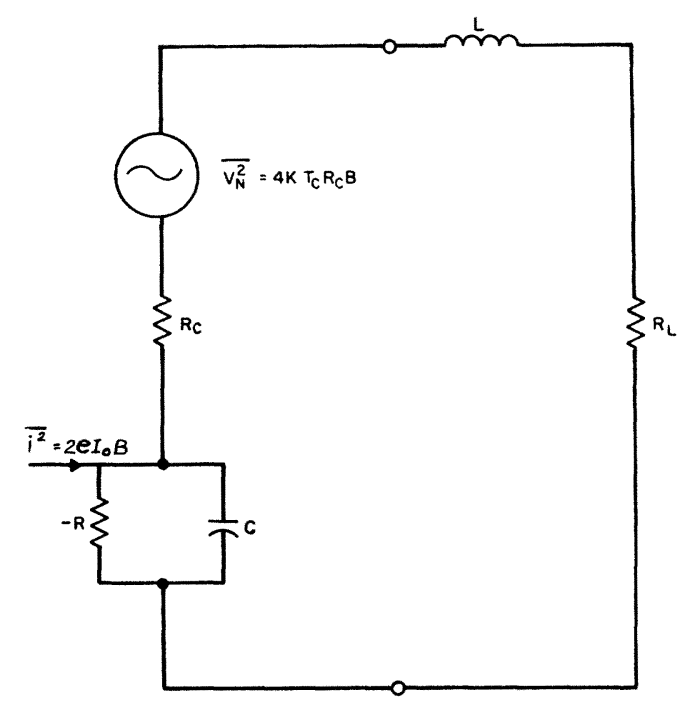

Fig. 3-The equivalent circuit of the Esaki diode amplifier.

which accounts for the barrier capacitance. The spreading and contact resistance of the diode are lumped together with the ohmic losses of the cavity in $R_{c}$. The noise generated by the three sources of loss listed above is provided by a Johnson noise generator of mean-square voltage amplitude,

$$
\overline{v^{2}}=4 k T_{c} R_{c} B,
$$

where $k$ is the Boltzmann constant and $T_{c}$ is the ambient temperature of the cavity. $L$ stands for the total inductance (parasitic and intentional). The shot noise generator

$$
\overline{i_{2}}=2 e I_{0} B
$$

was discussed above. The load resistance is $R_{L}$.

For the purpose of analysis, it is more convenient to transform the circuit to one in which all the elements are either in series or in parallel. The series equivalent circuit and the new expressions for the equivalent series circuit elements are shown in Fig. 4.

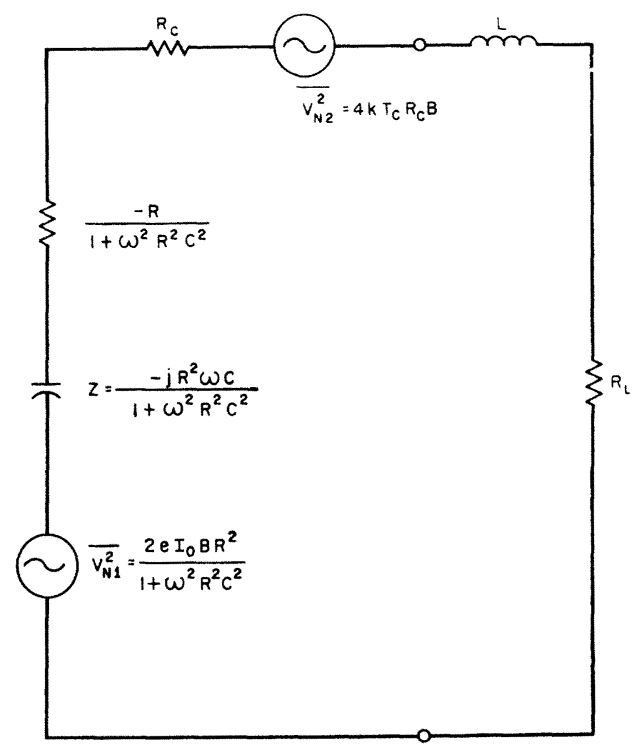

Fig. 4-The series equivalent circuit of the Esaki diode amplifier.

Series resonance occurs at

$$
\omega_{\mathrm{res}}=\omega_{0} \sqrt{1-\frac{1}{\omega_{0}^{2} R^{2} C^{2}}}
$$

where $\omega_{0}^{2}=1 / L C$. For resonance to occur at real frequencies, we must fulfill the condition

$$
\omega_{0} R C>1
$$

for $\omega_{0}^{2} R^{2} C^{2} \gg 1, \omega_{\text {res }} \approx \omega_{0}$.

A necessary and sufficient condition for positive gain is that the real part of the total series resistance, seen looking back into the amplifier terminals, be negative; or from Fig. 4,

$$
\frac{R}{1+\omega^{2} R^{2} C^{2}}>R_{c} \text {. }
$$

The highest frequency at which this can take place is called the cutoff frequency, $f_{c o}$ :

$$
f_{c o}=\frac{\sqrt{R / R_{c}-1}}{2 \pi R C}
$$

and represents the highest useful frequency for a given diode and circuit.

Since our main interest is in an amplifier operating at resonance, we evaluate the elements of the equivalent circuit of Fig. 4 for the condition $\omega=\omega_{\text {res. }}$. The result is shown in Fig. 5. The effective negative resistance is now represented by the series resistance

$$
R^{\prime}=\frac{-R}{\omega_{0}^{2} R^{2} C^{2}}
$$




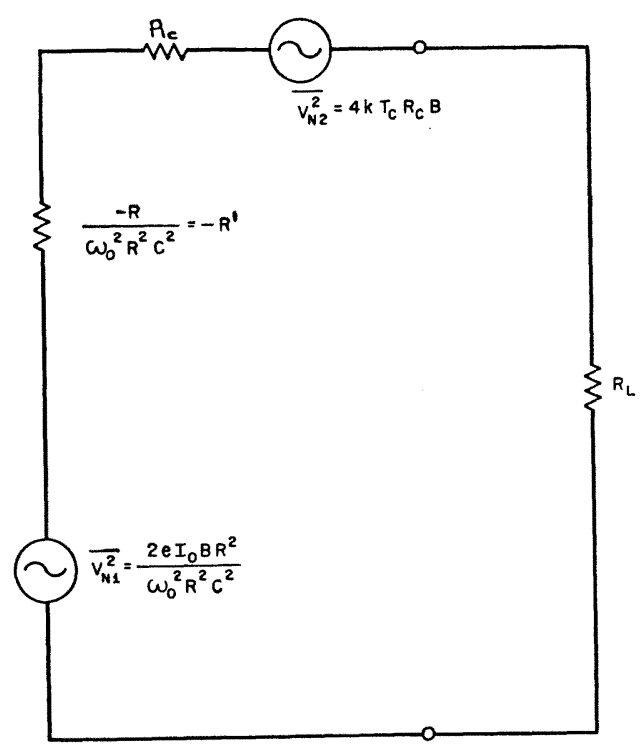

Fig. 5-The series equivalent circuit of the Esaki diode amplifier at resonance.

while the shot noise is now provided by a voltage generator of mean-square amplitude

$$
\overline{V_{N 1}^{2}}=\frac{2 e I_{0} B R^{2}}{\omega_{0}^{2} R^{2} C^{2}}
$$

The physical importance attached to the elements of the equivalent circuit of Fig. 5 is of paramount importance and contains a number of pitfalls, so that a few pertinent remarks are in order. Since the resistances are all in series, they are proportional to power, with the result that one may wrongly infer, for instance, that the power generated by the diode is proportional to $R$ and, therefore, that for large gain or large power output, diodes with large $R$ are superior. In actuality, the generated power is proportional to $1 / R$, which for a given diode material is proportional to the junction area. Physical significance can only be attached to ratios of any pair of resistances in Fig. 5. The quantity $R^{\prime} / R_{L}$ is equal to the ratio of power generated by the diode to that delivered to the load, while $R_{L} / R_{c}$, for instance, gives the ratio of the power consumed by the load to that expended in the cavity. $R_{L} / R_{c}$ is of ten referred to as the coupling ratio of the cavity. (Ratio of cavity " $Q$ " to external " $Q$ ".)

\section{The Gain-Bandwidth Product}

The power gain $G$ of the amplifier is defined as the ratio of the power delivered to the load (the reflected power in our case) to the power available from the source (incident) and is thus equal to the squared magnitude of the reflection coefficient measured at the amplifier terminals. Using Fig. 5, we have

$$
G=\left[\frac{R_{L}-\left(R_{c}-R^{\prime}\right)}{R_{L}+\left(R_{c}-R^{\prime}\right)}\right]^{2}
$$

The bandwidth $\Delta f$ is the distance, in cps, between the half-power frequencies. It is derived by replacing $R_{c}-R^{\prime}$ in (7) by the total series impedance away from resonance as given by Fig. 4 . The result is

$$
\Delta f=\frac{R_{L}+R_{c}-R^{\prime}}{2 \pi L\left(1-\frac{1}{\omega_{0}^{2} R^{2} C^{2}}\right)},
$$

yielding for the voltage gain-bandwidth product

$$
(\sqrt{G})_{\mathrm{res}} \Delta f=\sqrt{G} \Delta f=\frac{R_{L}+R^{\prime}-R_{c}}{2 \pi L\left(1-\frac{1}{\omega_{0}^{2} R^{2} C^{2}}\right)} .
$$

Eq. (8) takes a meaningful form upon substitution of the high gain condition ${ }^{9}$

$$
R^{\prime} \approx R_{c}+R_{L}
$$

and the definition of $R^{\prime}$ as given by (6). The result is

$$
(\sqrt{G} \Delta f)_{\mathrm{high} \mathrm{gain}}=\frac{1-R_{\mathrm{c}} / R^{\prime}}{\pi R C\left(1-\frac{1}{\omega_{0}^{2} R^{2} C^{2}}\right)},
$$

in which form it is amenable to experimental verification.

To increase the gain-bandwidth product, we have to use a diode with a small $(R C)$. This happens, not only through the dependence of $\sqrt{G} \Delta f$ on $(R C)$ as given by (10), but also through a less obvious decrease of $R_{c} / R^{\prime}$. If we assume that the total inductance has been made as small as possible, operation at one frequency means using the same $C$. If one now goes to a diode material with an intrinsically smaller $(R C)$ product, it becomes possible to operate at the same frequency with a diode having a smaller $R,{ }^{10}$ i.e., a diode generating more power, which corresponds to a smaller $R_{c} / R^{\prime}$ ratio.

\section{The Noise Temperature of the Amplifier}

The noise temperature $T_{e}$ of the amplifier, ${ }^{11}$ which is sometimes called the effective input noise temperature, can be defined as the increase in the source temperature which is required to keep the noise power output a constant if the amplifier, hypothetically, were rendered noiseless. It is found by equating that part of the total noise output originating within the amplifier, $N_{A}$, to $k T_{e} G B$, i.e.,

$$
N_{A}=k T_{e} G B
$$

- This condition, when taken with an equality sign, makes the gain infinite [see (7)] and becomes the "start-oscillation" condition.

10 'The new diode will have the same area as the old one, but will possess a larger current density and, consequently, a larger current.

${ }_{11} T_{e}$ is related to the noise figure $F$ by

$$
F=1+\frac{T_{e}}{290} .
$$


or using the equivalent circuit of Fig. 5 and the gain definition of $(7)$,

$$
\begin{aligned}
k T_{e} B & \frac{\left(R_{L}-R_{c}+R^{\prime}\right)^{2}}{\left(R_{L}+R_{c}-R^{\prime}\right)^{2}}=4 k T_{c} B \frac{R_{c} R_{L}}{\left(R_{L}+R_{c}-R^{\prime}\right)^{2}} \\
& +\frac{2 e I_{0} B R R^{\prime} R_{L}}{\left(R_{L}+R_{c}-R^{\prime}\right)^{2}}
\end{aligned}
$$

where $T_{c}$ is the temperature of the cavity and diode. Solving for $T_{e}$ yields

$$
T_{e}=\frac{4 T_{c} R_{c} R_{L}}{\left(R_{L}-R_{c}+R^{\prime}\right)^{2}}+\frac{2 e I_{0} R R^{\prime} R_{L}}{k\left(R_{L}-R_{c}+R^{\prime}\right)^{2}}
$$

which can be transformed to

$$
T_{e}=\frac{(\sqrt{G}+1)^{2}}{G}\left[T_{c}\left(\frac{R_{c}}{R_{L}}\right)+\frac{e I_{0} R}{2 k}\left(\frac{R^{\prime}}{R_{L}}\right)\right],
$$

in which form only ratios of resistance appear. This form is particularly useful, since in it the dependence of the noise temperature on the gain is separated from its dependence on the coupling ratios. This bracketing is justified operationally, since the same value of gain can be obtained with different combinations of coupling ratios.

\section{Experimental Results}

The pertinent characteristics of the phosphorusdoped germanium diode were:

$$
\begin{aligned}
I_{\text {peak }} & =1 \mathrm{ma} \\
I_{0} R & =8.6 \times 10^{-2} \text { at the biasing point } \\
R C & \cong 3.5 \times 10^{-10} \pm 10 \text { per cent } \\
R & =175 \text { ohms. }
\end{aligned}
$$

The diode was mounted between the ridge and top wall of a short-terminated waveguide, the distance between the diode and the short being adjustable for tuning purposes. The ridge waveguide tapered gradually into a 2 -inch $\times 1$-inch input waveguide which was coupled to the input and output via a low-loss circulator. The diode coupling was controlled by two screws placed, respectively, $\frac{1}{8}$ and $\frac{3}{8}$ guide wavelengths in front of the diode. Fig. 6 shows a photograph of the ridge waveguide cross section at the diode position, while Fig. 7 shows, with considerable magnification, the immediate area surrounding the diode.

The measured gain-bandwidth product at $4500 \mathrm{Mc}$ was $3.6 \times 10^{8}$, and was checked at a number of gain settings between $17 \mathrm{db}$ and $25 \mathrm{db}$, where it was found to be a constant, in agreement with (10). The gainbandwidth product deteriorated for lower gain settings.

The measured noise temperature was $1740^{\circ} \mathrm{K}$ and was taken with the amplifier set for a gain of $25 \mathrm{db}$. To compare this result with that predictable from (8), it is necessary to know the power ratios $R_{c} / R_{L}$ and $R^{\prime} / R_{L}$. This was accomplished by using the measured values of

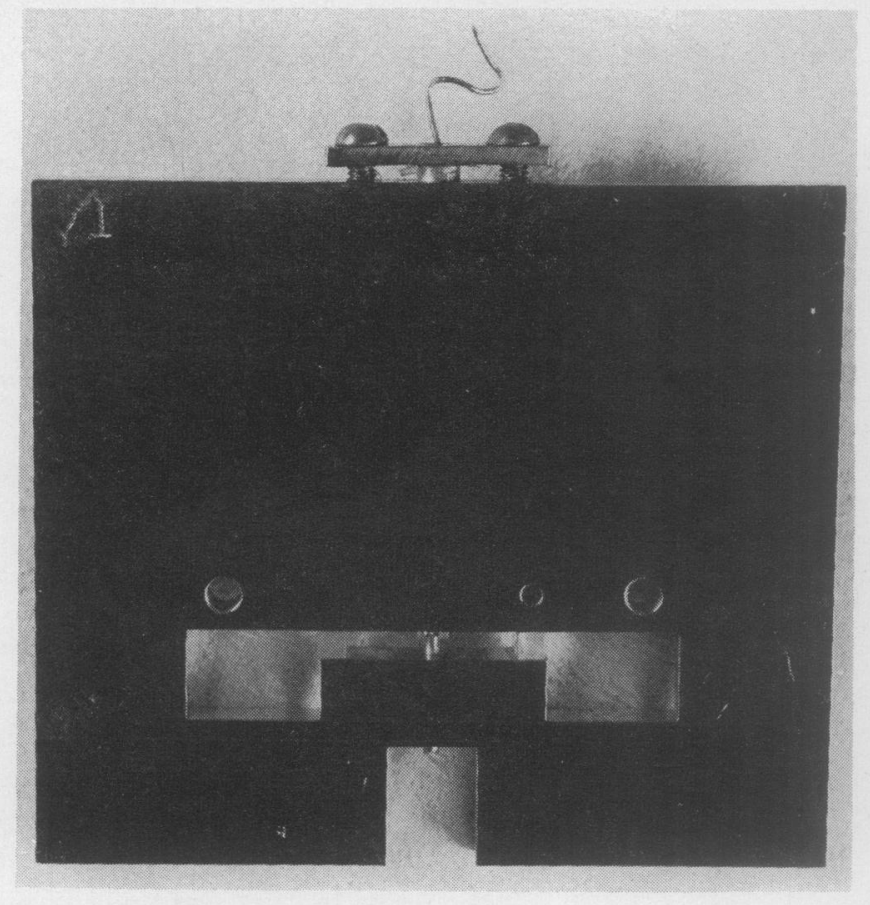

Fig. $6-$ Cross section of the ridge waveguide at the diode position.

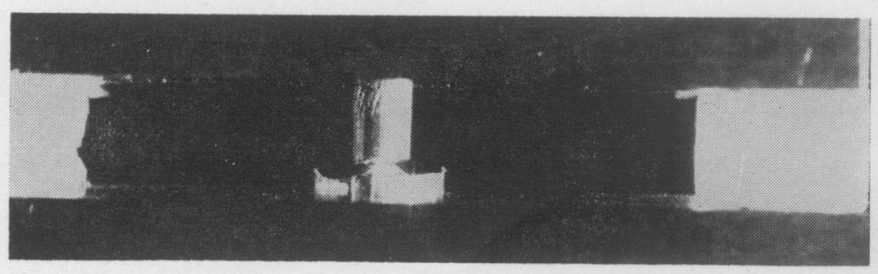

Fig. 7-An enlarged view of the diode and its surroundings. The diode is mounted between the two pins at the center of the photograph.

$\sqrt{G} \Delta f$ and $(R C)$ to solve for $R_{c} / R^{\prime}$ in (10), and then by using the high gain condition (9) to solve for the required ratios. With the numerical data given above, the ratios are:

$$
\frac{R_{c}}{R^{\prime}}=0.6, \quad \frac{R^{\prime}}{R_{L}}=2.5, \quad \frac{R_{c}}{R_{L}}=1.5 .
$$

Substitution in (11) yields

$$
\left(T_{e}\right)_{\text {calculated }}=1890^{\circ} \mathrm{K} \pm 10 \text { per cent, }
$$

which is to be compared with the experimental value of $1740^{\circ} \mathrm{K}$. The main source of error is the uncertainty in $(R C)$. The satisfactory agreement between the experimental and theoretical results serves as an indirect check on the existence of full shot noise at microwave frequencies in tunnel diodes. A more recent version of the same amplifier yielded a noise temperature of $1200^{\circ} \mathrm{K}$.

\section{Concluding Remarks}

A number of factors which are evident from (11) affect the noise temperature. We shall treat them separately. 
The dependence of $T_{e}$ on the gain $G$ is contained in the factor $(\sqrt{G}+1)^{2} / G$, which decreases asymptotically toward unity with increasing gain so that high gain operation is imperative.

The shot noise contribution enters through the term $I_{0} R$. It can be minimized by optimum biasing of the diode in such a manner that the product $I_{0} R$ is a minimum. In practice, it was found impractical to operate the diode for any considerable distance beyond the midrange of the negative resistance, because the increasing value of $R$, due to the curvature of the $V$ - $I$ curve, more than offset the decrease in $i_{0}$. The problem of the exact shape of the $V-I$ curve is intimately related to that of the excess current ${ }^{12,13}$ and has not been settled yet.

The $\left(I_{0} R\right)$ product is inherently smaller for semiconductors with small energy gaps ( $\mathrm{InSb}-0.17 \mathrm{v}, \mathrm{InAs}-$ $0.33 \mathrm{v}, \mathrm{Ge}-0.72 \mathrm{v}$ ) which should therefore be preferred for low noise applications. This advantage is offset to some extent by the fact that, assuming the same $(R C)$ product, the high-energy-gap diodes generate more power and thus make it easier to overcouple the cavity and still achieve high gain.

The shot noise contribution to the noise temperature is proportional to $R^{\prime} / R_{L}$, which, according to (9), is always larger than unity. In the limit when the cavity losses are very small compared to the generated power and the load power, i.e., when $R_{c} / R^{\prime} \approx R_{c} R_{L} \ll 1$, the ratio $R^{\prime} / R_{L}$ approaches unity. As pointed out at the conclusion of Section III, this situation is approached, for a diode operating at a fixed frequency, by using diodes with small values of $(R C)$. The shot noise contribution to the amplifier's noise temperature is thus always larger, but hopefully not much larger, than $e I_{0} R / 2 k$. In our amplifier this was $\sim 500^{\circ} \mathrm{K}$.

The same conditions that minimize the shot noise contribution also cause the ratio $R_{c} / R_{L}$ to be a minimum, thus minimizing the contribution of the cavity losses at $T_{c}$ [see (11)].

12 R. A. Logan and A. G. Chynoweth, "Effect of radiation damage on excess current in Esaki diodes," Bull. Am. Phys. Soc., vol. 5, series II, p. 375; June 15, 1960.

${ }_{13}$ T. Yajima and L. Esaki, "Excess noise in narrow germanium p-n junctions," J. Phys. Soc. Japan, vol. 13, pp. 1281-1287; November, 1958.
The reduction of $(R C)$ has a beneficial effect on both the gain-bandwidth product and the noise temperature of the amplifier; it also creates a number of problems. The first is one of stability. For stable operation we must satisfy the condition ${ }^{14}$

$$
L(\omega)<R_{s}(\omega) R C
$$

at all frequencies. Where $L(\omega)$ and $R_{s}(\omega)$ are, respectively, the total inductance and resistance in series with the diode, they are shown as functions of frequency since stable operation has to obtain for all frequencies. The difficulty of satisfying the stability condition increases with smaller $(R C)$ product.

A small value of $R C$ makes $R$ smaller, at a given frequency, and entails using a microwave structure with low characteristic impedances. In the diode used in our experiment, $R \sim 175 \mathrm{ohms}$ and $R C \sim 3.5 \times 10^{-10}$. Had we used instead a unit with $R C \sim 10^{-11}$, which is available, the negative resistance $R$ would have been $\sim 5 \mathrm{ohms}$, which is a very low impedance level in microwave circuitry and creates a gamut of problems, such as matching to standard components with much higher impedances and the high attenuation of low-impedance waveguides.

It is not likely that tunnel diode will ever threaten the maser and parametric amplifiers as a low-noise amplifier. It may still find application in cases where its extreme simplicity and economy may be traded for increased noise, especially when tunnel-diode amplifiers with noise figures substantially lower than the one reported above will be made. This improvement is mainly contingent on the utilization and taming of diodes with small $(R C)$ products and on the use of low-energy-gap diodes - with small $\left(I_{n} R\right)$ products.

\section{ACKNowledGMENT}

The authors wish to express their indebtedness to P. E. Butzien for his able participation in the experimental work, and to E. Dickten for fabricating and mounting the diodes used in the experiment.

\footnotetext{
${ }^{14}$ R. L. Wallace, unpublished report.
} 